\title{
Grammar in art
}

\section{Edward Segel and Lera Boroditsky*}

Department of Psychology, Stanford University, Stanford, CA, USA

\section{Edited by:}

Asifa Majid, Max Planck Institute for Psycholinguistics, Netherlands

\section{Reviewed by:}

Andrea Bender, University of Freiburg,

Germany

Dan Isaac Slobin, University of

California Berkeley, USA

*Correspondence:

Lera Boroditsky, Department of

Psychology, Jordan Hall, Building

01-420, Stanford University, 450 Serra

Mall, Stanford, CA 94305, USA.

e-mail: lera@psych.stanford.edu
Jakobson (1959) reports: "The Russian painter Repin was baffled as to why Sin had been depicted as a woman by German artists: he did not realize that 'sin' is feminine in German (die Sünde), but masculine in Russian (rpex)." Does the grammatical gender of nouns in an artist's native language indeed predict the gender of personifications in art? In this paper we analyzed works in the ARTstor database (a digital art library containing over a million images) to measure this correspondence. This analysis provides a measure of artists' real-world behavior. Our results show a clear correspondence between grammatical gender in language and personified gender in art. Grammatical gender predicted personified gender in $78 \%$ of the cases, significantly more often than if the two factors were independent. This analysis offers a new window on an age-old question about the relationship between linguistic structure and patterns in culture and cognition.

Keywords: language, thought, grammatical gender, art

\section{INTRODUCTION}

Jakobson (1959) reports: “The Russian painter Repin was baffled as to why Sin had been depicted as a woman by German artists: he did not realize that 'sin' is feminine in German (die Sünde), but masculine in Russian (rpex)." Does the grammatical gender of nouns in an artist's native language indeed predict the gender of personifications in art? While this question has been considered and debated by art scholars (e.g., Guthke, 1999), no quantitative analysis has been offered. In this paper we analyzed a large art database to measure this correspondence. This analysis offers a new window on an age-old question about the relationship between linguistic structure and patterns in thought (e.g., Whorf, 1956).

Previous work has examined the role of grammatical gender in linguistic processing and semantic judgments in laboratory tasks (e.g., Konishi, 1993; Sera et al., 1994; Flaherty, 2001; Vigliocco et al., 2004). Here we take a different approach and consider a pattern of behavior in the real world: personification in art. While observational approaches like this are necessarily limited for the purposes of inferring causation, observing real-world behavior does offer a number of important advantages.

In many laboratory tasks, participants may guess the purpose of the study. For example, if explicitly asked whether an apple is more similar to a man or a woman, a Spanish speaker may guess that the study is about grammatical gender and respond accordingly. Our "participants" could not have been aware of the purpose of the study since most were no longer alive when the experiment was conceived. A further worry about effects observed in the laboratory is that they might be hot-house flowers. That is, such effects may be coaxed into existence only under perfectly controlled laboratory conditions but are too weak to have import in the real world where they are easily overwhelmed by more powerful forces. The present method measures people's behavior outside of the laboratory in the full complexity of the real world. A final worry is that laboratory findings may reveal only momentary biases, inconsequential short-term perturbations that people immediately overcome if making any real decisions in their lives. In this case, our objects of study are artworks that took their creators weeks, years, or decades to complete, requiring extended effort and commitment and allowing plenty of time for reasoned reflection.

Finally, the behavioral measure is non-linguistic: the objects of study are paintings, drawings, and sculptures. In cross-linguistic comparisons of behavior, observations from non-linguistic tasks are important to establish that patterns exist in how people think and not just in how they talk.

\section{MATERIALS AND METHODS}

We started with all works of art contained in the ARTstor database, a digital library of more than one million images. From this database, all images from $1200 \mathrm{AD}$ to present day that were indexed by the keywords "personification" or "allegory" were selected. This yielded 2749 results from a diverse range of cultural and artistic heritages. For tractability, the analysis was restricted to artworks from Italy, France, Germany, and Spain (where the languages spoken have grammatical gender, and for which a large-enough sample of artworks existed in the database). This yielded 1708 images for analysis, or $62 \%$ of the total.

Each of the 1708 images was analyzed for usability in the study. The following categories of images were excluded. Three hundred eighty-four images were duplicated in the sample, and so the duplicates were removed. Three hundred fifty-five were allegories that did not include a personification of an abstract entity (e.g., Caravaggio's "Beheading of St. John the Baptist" showing St. John the Baptist). For 151, the allegory was unclear (the title or image did not clearly designate an allegory). In 67 it was unclear which of the depicted humans represented the abstract entity, in 62 the depiction was non-human (e.g., animal, inanimate object), in 40 the gender of the personification was unclear, and in 33 the personification was in the form of a mixed gender group. Excluding the images listed above yielded 616 images. These images contained 790 clear personifications (106 images contained more than one personification), and each personification 
could be clearly classified as a female or male depiction. All of the judgments described above were carried out by a naïve coder, not familiar with the grammatical genders of nouns in the languages of interest.

The cases were then categorized according to the artist's native language (422 Italian, 213 French, 129 German, and 24 Spanish, 2 Dutch), and the grammatical gender for each depicted allegory was determined. Artists' native languages were ascertained using available biographical information. In cases where no biographical information was available or in cases of ambiguity or works for which the artist is unknown, the native language was coded in accordance with the country of origin listed for the image in the ARTstor database. The standard grammatical genders of nouns were determined by consulting dictionaries and translation engines. We note that especially for early works, it is possible that grammatical genders (and even grammatical gender systems as is the case with Old English) used at the time were different from modern usage. Such changes would only add noise to the data set and preclude the analysis from seeing clear patterns. To make a conservative estimate the analysis relied purely on modern grammatical genders. Two of the works were produced by Dutch artists (not one of the target languages in the study) and 23 of the German personifications were in the grammatically neuter category and so were not included in further analyses. This yielded 765 cases to be included in the main analysis of interest.

\section{RESULTS}

Overall, the depicted gender matched the grammatical gender in $78 \%$ of the cases, significantly more often than if the two factors were independent, $\chi^{2}(1, N=765)=172.7, p<0.00001$; oddsratio $=9.33$. Grammatically feminine entities were more likely to be personified as female ( $86 \%$ female, $14 \%$ male), and grammatically masculine entities were more likely to be personified as male (40\% female, $60 \%$ male). See Table 1 .

We analyzed the data in a number of different ways to control for non-independence of personifications that occurred within the same artwork, that were produced for the same allegorical theme, or that were produced by the same artist. Excluding all images that contained multiple personifications yielded the same pattern: depicted gender agreed with grammatical gender $76 \%$ of the time, $\chi^{2}(1, N=490)=85.2, p<0.00001$. A by-allegory analysis likewise showed the same pattern: depicted gender agreed with grammatical gender $72 \%$ of the time, $\chi^{2}(1, N=243)=32.9, p<0.00001$. The pattern also held in a by-artists analysis: depicted gender agreed with grammatical gender $75 \%$ of the time, $\chi^{2}(1, N=234)=28.0$, $p<0.00001$. Finally, we constrained our analysis to only those allegories for which our sample contained examples with conflicting

Table 1 | Number of female and male personifications shown by grammatical gender.

\begin{tabular}{lcc}
\hline & \multicolumn{2}{c}{ Grammatical gender } \\
\cline { 2 - 3 } & Feminine & Masculine \\
\hline Personified as female & 454 & 94 \\
Personified as male & 74 & 143
\end{tabular}

grammatical genders. The pattern still held: depicted gender agreed with grammatical gender $71 \%$ of the time, $\chi^{2}(1, N=217)=36.7$, $p<0.00001$.

The same pattern of results was present across the four languages included in the study: $82 \%$ of personifications by Italian artists matched Italian grammatical gender, $80 \%$ for French, $62 \%$ for German, and 54\% for Spanish (though note that our sample includes only 24 Spanish cases, the lowest number among the languages included by far).

There were many more female personifications than male overall ( $71.6 \%$ or 548 out of 765 were female), possibly revealing an overall bias for depicting the female form among the artists in our sample.

The sample we analyzed included personifications of 243 distinct entities, including (in order of frequency) love, justice, time, charity, fame, fortune, peace, truth, and many more. Clearly, for the very common allegorical themes there may exist strong precedents from history, mythology, and cultural tradition that can shape the artist's choice in personification. However, there is less precedent for uncommon allegories (geometry, necessity, silence). To test whether the effects we observe are driven only by the most common allegories, we divided the allegories into the most common (30 allegories representing 385 cases), and the least common (213 allegories representing the remaining 380 cases). The least common allegories still independently showed strong congruence with grammatical gender $\left[75 \% ; \chi^{2}(1, N=380)=60.2, p<0.00001\right]$, as did the most common allegories $\left[81 \% ; \chi^{2}(1, N=385)=124.3, p<0.00001\right]$.

\section{DISCUSSION}

Our results show a clear correspondence between grammatical gender in language and personified gender in art. Of course, grammatical gender is only one of the forces that may influence an artist's decisions in personification. Take the example of Alfred Kubin, a Czech-born and Austrian-educated expressionist whose drawing "The best physician" portrays death as a woman. Kubin's mother died when he was 10, he attempted to commit suicide on her grave at 19 , and a few years later suffered a nervous breakdown after the death of his fiancé. It is quite clear that the grammatical gender of "death" in Czech (feminine) or German (masculine) would not have been the only source of influence in forming Kubin's conception of death. Considering how many different forces can exert influence on an individual's conceptualization of abstract entities like love and death, it seems especially striking that grammatical gender - a small quirk of grammar - can be used to predict the gender of personification $78 \%$ of the time.

Of course, artists do not produce their work in a historical vacuum. For many allegorical themes there are strong precedents from history, mythology, and cultural tradition that can shape the artist's choice in personification. We do find, however, that even for uncommon allegories (geometry, necessity, silence) where less historical precedent is available, there is still a strong correspondence with grammatical gender. In a correlational analysis like this it is not possible to establish whether grammatical gender in language helped create the extra-linguistic cultural patterns we observe or whether the influence went the other way, or both. It seems likely that linguistic and extra-linguistic aspects of culture act in concert and mutually reinforce each other, in many reciprocal cycles of 
causation over time. Structures in language may influence artistic decisions, and in turn patterns in artistic tradition may encourage, enrich, or help reify structures in language, and so on.

In our analysis we considered the correspondence between personification in visual art and grammatical gender in French, Italian, Spanish, and German. Extending this investigation to other languages and new language groups may offer interesting comparisons to the current data. Would the patterns still hold in languages that have more than two or three genders? Would the patterns hold beyond Europe, in a different set of artistic and cultural traditions? Beyond paintings and sculptures, personification is also abundant in poetry, fairy tales, plays, and novels, as well as in films, animations, music, and dance. It would be interesting to examine the patterns of correspondence in personification across these different forms of expression, as well as any further cultural consequences of such personification.

\section{REFERENCES}

Flaherty, M. (2001). How a language gender system creeps into perception. J. Cross Cult. Psychol. 32, 18-31.

Guthke, K. S. (1999). The Gender of Death: A Cultural History in Art and Literature. Cambridge: Cambridge University Press.

Jakobson, R. (1959). “On linguistic aspects of translation," in On Translation, ed. R. A. Brower (Cambridge, MA: Harvard University Press), 232-239.

Konishi, T. (1993). The semantics of grammatical gender: a cross-cultural study. J. Psycholinguist. Res. 22, 519-534.

Sera, M. D., Berge, C., and del Castillo, J. (1994). Grammatical and conceptual forces in the attribution of gender by English and Spanish speakers. Cogn. Dev. 9, 261-292.

Vigliocco, G., Vinson, D., Indefrey, P., Levelt, W., and Hellwig, F. (2004). Role of grammatical gender and semanticsin German word production. J. Exp. Psychol. Learn. Mem. Cogn. 30, 483-497.

Whorf, B. (1956). Language, Thought, and Reality: Selected Writings of Benjamin

\section{CONCLUSION}

We examined the personified gender of abstract entities in art. The analysis revealed that a small quirk of grammar (the grammatical assignment of a noun as masculine or feminine) could be used to predict the gender of personification in art $78 \%$ of the time. This analysis demonstrates a close coupling between grammatical structures in language and other aspects of culture. It appears that patterns in language may not only be reflected in our private mental lives, but may also become reified in the material world we create around us.

\section{ACKNOWLEDGMENTS}

This research was funded by NSF Grant No. 0608514 to Lera Boroditsky. We thank Claire Schmauch for preliminary data coding and analyses.

Lee Whorf, ed. J. B. Carroll. Cambridge, MA: MIT Press.

Conflict of Interest Statement: The authors declare that the research was conducted in the absence of any commercial or financial relationships that could be construed as a potential conflict of interest.

Received: 20 October 2010; paper pending published: 08 November 2010; accepted: 24 December 2010; published online: 13 January 2011.
Citation: Segel E and Boroditsky L (2011) Grammar in art. Front. Psychology 1:244. doi: 10.3389/fpsyg.2010.00244

This article was submitted to Frontiers in Cultural Psychology, a specialty of Frontiers in Psychology.

Copyright (c) 2011 Segel and Boroditsky. This is an open-access article subject to an exclusive license agreement between the authors and the Frontiers Research Foundation, which permits unrestricted use, distribution, and reproduction in any medium, provided the original authors and source are credited. 